DIVISION OF THE HUMANITIES AND SOCIAL SCIENCES

CALIFORNIA INSTITUTE OF TECHNOLOGY

PASADENA, CALIFORNIA 91125

VIRTUAL REPEATED IMPLEMENTATION

Christopher P. Chambers

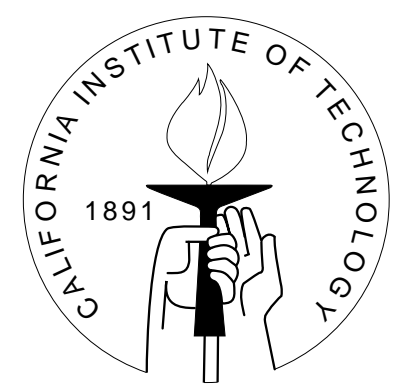

SOCIAL SCIENCE WORKING PAPER 1179

September 2003 


\title{
Virtual Repeated Implementation
}

\author{
Christopher P. Chambers
}

\begin{abstract}
We show that in the context of repeated implementation, any social choice rule which realizes all alternatives for a positive (yet arbitrarily small) amount of time is Nash implementable. The results complement those of the virtual implementation literature.
\end{abstract}

JEL classification numbers: C72, D60

Key words: virtual implementation, repeated implementation 


\title{
Virtual Repeated Implementation
}

\author{
Christopher P. Chambers*
}

\section{Introduction}

A social planner desires to implement some social policy or alternative for society but does not know the preferences of the agents. Implementation theory focuses on methods of eliciting these preferences in various contexts. The typical way is to create an incentive for agents to announce their preferences. This is done is by forcing agents to play a game, in which by acting in their best interests, they end up implementing the social choice rule. The main question of the implementation literature is which types of social choice rules can be so implemented. Our purpose is to discuss a connection between two strands of the implementation literature.

Repeated implementation focuses on the specific case in which the social alternative is actually a stream of alternatives-that is, the social alternative may change through time. Suppose agents possess time-separable preferences, and all agents' discount factors are known to the social planner. Kalai and Ledyard [3] introduce this model and establish important results on implementation in this environment. They establish that for any given social choice rule, one can construct another social choice rule which coincides with the original one after some fixed period of time and which is implementable in dominant strategies. Thus, it is always in all agents best interest to report their true preferences, irrespective of what other agents do.

Virtual implementation focuses on the special case of risky social alternatives (i.e. lotteries) and agents who conform to the expected utility axioms. In this one-stage environment, any social choice rule which, for every possible preference profile, assigns a positive probability to all social alternatives can be implemented in Nash equilibrium (i.e. there exists a game form for which agents playing a Nash equilibrium select the desired social alternative).(see Matsushima [6] and Abreu and Sen [1]).

We exploit the similarity of the linear structure of time separable preferences and expected utility preferences to establish a result closely related to the virtual implementation result described above. Working in a continuous time repeated implementation

*Assistant Professor of Economics, Division of the Humanities and Social Sciences, 228-77, California Institute of Technology, Pasadena, CA 91125. Email: chambers@hss.caltech.edu. Phone: (626) 3953559. I would like to thank John Ledyard for comments and suggestions. All errors are my own. 
framework, we establish that any social choice rule which, for every possible preference profile, realizes each social alternative for a positive (yet arbitrarily small) amount of time, is Nash implementable. Thus, we may think of our result as a reinterpretation of the virtual implementation results that might be called "repeated virtual implementation."

Our main result relies on the critical assumption that the social planner knows each agents' discount factor. If this is not the case, almost nothing is implementable in our environment. This assumption should not be taken lightly. We point out; however, that the assumption of known discount factors is also present in the work of Kalai and Ledyard [3], where they play an even more critical role. In our work, any social choice rule which realizes all alternatives is Nash implementable. In their work, the class of social choice rules which are implementable (in dominant strategies) is a function of the discount factors of the agents; when agents are more patient, less social choice rules are implementable.

The other critical assumption is that preferences are time-separable. Linearity assumptions on preference have been criticized in the literature (see, for example, Jackson [2]). Linearity is usually assumed to obtain tractability of a model. However; virtual implementation results are not robust to the specification of linear utility. For example, in the risk case, an agent who has non-expected utility preferences does not have linear preferences. In the repeated implementation case, general recursive utility preferences (as in Koopmans [4]) are non-linear.

Section 2 provides the model. Section 3 presents our main result. Section 4 concludes.

\section{The model}

Let $N$ be a set of agents, where $|N| \geq 3$. Let $X$ be a finite set of alternatives. Alternatives are meant to be understood as social alternatives, which may be realized at a given point in time. The set of alternative streams is the set of functions $\bar{x}:[0, \infty) \rightarrow X$ which are Borel measurable; $X^{*}$ denotes the set of alternative streams. Thus, our model is one of continuous time, where time is indexed from 0 to $\infty .^{1}$ An alternative stream is interpreted as a social alternative which changes over time. There are many reasons for allowing a social alternative to change over time; the most obvious such reason is to achieve fairness (for example, selecting my favorite alternative today and yours tomorrow).

Agents' preferences over alternative streams are representable by time-separable utility functions. We assume that the social planner knows each agents' discount factor. To this end, for all $i \in N$, let $\delta_{i} \in(0,1)$ be a discount factor for agent $i$. For all $i$, let $\mathcal{U}_{i}$ be a collection of functions $u_{i}: X \rightarrow \mathbb{R}$ which are nonconstant. The set $\mathcal{U}_{i}$ is interpreted as a set of possible utility indices. Agent $i$ 's preference over alternative streams

\footnotetext{
${ }^{1}$ All of our results continue to hold if time ends at some $T>0$.
} 
is represented by $U_{i}: X \rightarrow \mathbb{R}$, where $U_{i}(\bar{x})=\int_{0}^{\infty} e^{-\delta_{i} t} u_{i}(\bar{x}(t)) d t$, for some $u_{i} \in \mathcal{U}_{i}$. The set of preference relations for agent $i$ is denoted by $\mathcal{R}_{i}$. Given that each agent's discount factor is known, a utility index uniquely identifies a preference over alternative streams.

Say an alternative stream $\bar{x}$ realizes all alternatives if for all $x \in X$, $\int_{0}^{\infty} 1_{\{\bar{x}(t)=x\}} d t>0$. Thus, an alternative stream realizes all alternatives if every possible alternative is in place for some positive (perhaps arbitrarily small) amount of time. The set of alternative streams which realize all alternatives is denoted by $X^{r}$.

Define a social choice rule as a nonempty-valued correspondence $\psi: \prod_{i \in N} \mathcal{R}_{i} \rightrightarrows$ $X^{*}$. A social choice rule recommends a collection of alternative streams for every possible profile of utility indices. Say a social choice rule $\psi$ realizes all alternatives if for all $R \in \prod_{i \in N} \mathcal{R}_{i}, \psi(R) \subset X^{r}$.

We assume that the social planner does not know the agents' preferences over alternative streams. Thus, he must rely on the agents to announce their preferences. We study the reliability of such announcements. Hence, define a game form as a tuple $(S, g)$, where $S \equiv \prod_{i \in N} S_{i}$, and $g: S \rightarrow X^{*}$. The set $S$ is a set of strategies. Given a preference profile $R=\left(R_{i}\right)_{i \in N}$, a game form naturally induces a normal-form game, where $s$ is preferred to $s^{\prime}$ by agent $i$ if $g(s) R_{i} g\left(s^{\prime}\right)$. Let $N(S, g, R)$ be the set of Nash equilibria of the game induced by $(S, g)$ and $R$. Say a social choice rule $\psi$ is Nash implementable if there exists a game form $(S, g)$ such that $g(N(S, g, R))=\psi(R)$. Thus, a social choice rule is Nash implementable if the social planner can set up a strategic situation whereby agents, playing the game in their best interests, select the recommended social alternative.

\section{Results}

The main result of this note is that any social choice rule which realizes all alternatives is Nash implementable.

Theorem: If a social choice rule realizes all alternatives, then it is Nash implementable.

The proof strategy is simple and is essentially a reinterpretation of virtual implementation $[1,6]$. Any alternative stream which realizes all alternatives can be thought of as being in the "interior" of the set of alternative streams. Maskin's famous theorem [5] asserts that any social choice rule which is "monotonic" and satisfies "no-veto power" is Nash implementable (when there are at least three agents). Given the "linear" structure of time-separable utility functions, the only Maskin monotonic transformations of a timeseparable preference in the "interior" of the set of alternative streams is that preference itself. Hence, such social choice rules are trivially monotonic. Any two preferences which coincide on the set of alternative streams which realize all alternatives coincide on all alternative streams. This fact allows us to construct an induced social choice rule 
defined on the domain of alternative streams which realize all alternatives, and timeseparable preferences restricted to this domain. On such a domain, no agent ever has a "most-preferred" alternative. Hence, no veto power is vacuously satisfied. Thus, at least on this domain, any social choice rule is implementable. The proof concludes by identifying a social choice rule over alternative streams with its induced social choice rule over alternative streams which realize all alternatives.

The theorem can be interpreted as saying that for any social choice rule, there exists a Nash implementable social choice rule which is "arbitrarily close" to the original rule. Agents must only wait through all alternatives at some point-perhaps only for a fleeting moment. It is not even specified when the agents must wait through these alternatives. This seems a small price to pay for completely resolving strategic issues.

Jackson [2] points out the possibility of renegotiation in the implementation literature. Such criticisms clearly apply in repeated implementation. Specifically, in our environment, suppose that alternative $x \in X$ is the least preferred by all agents according to the utility indices. A social choice rule which realizes all alternatives must feature $x$ for some positive amount of time. It seems natural for all agents to agree to "contract out" of this $x$ phase, as $x$ is Pareto dominated.

Kalai and Ledyard's [3] result focuses on those social choice rules which are almost dominant strategy implementable. Specifically, they show that for any social choice rule, there exists an alternative social choice rule which coincides with it after some point in time, and which is dominant strategy implementable. The major drawback of this work is that the amount of time that the agents need to wait until the desired rule is realized may be very large; in fact it is a function of the agents' discount rates. Agents may also only announce from a finite set of possible preferences. For all agents, these preferences must be ordinally distinct, in that utility indices cannot be monotonic transformations of each other. Our work has neither of these features. However; we restrict to a finite number of possible alternatives and require that the number of agents is at least three.

The proof is essentially a translation of the virtual implementation arguments into the repeated implementation framework. For a good intuition behind these types of proofs, see Jackson [2].

Proof: The proof is completed in a series of steps. Let $\psi$ be a social choice rule which realizes all alternatives. We show that $\psi$ is Nash implementable. For agent $i$, say that a preference relation $\mathbf{R}_{i}^{\prime}$ is a monotonic transformation of $\mathbf{R}_{i}$ at $\overline{\mathbf{x}}$ if for all $\bar{y}$ in the domain, $\bar{x} R_{i} \bar{y} \Longrightarrow \bar{x} R_{i}^{\prime} \bar{y}$. Say a rule $\psi$ is Maskin monotonic (see Maskin [5]) if whenever $\bar{x} \in \psi(R)$, if $R_{i}^{\prime}$ is a monotonic transformation of $R_{i}$ at $\bar{x}$ for all $i$, then $\bar{x} \in \psi\left(R^{\prime}\right)$. We will apply Maskin's famous theorem on sufficient conditions for implementation. Thus, say that a rule $\psi$ satisfies no veto power if there exists $i \in N$ such that if there exists $\bar{x}$ such that for all $j \neq i$, for all $\bar{y}, \bar{x} R_{j} \bar{y}$, then $\bar{x} \in \psi(R)$. Maskin shows that any rule satisfying Maskin monotonicity and no veto power is Nash implementable when there are at least three agents. 
Our proof establishes that any rule whose outcomes lie in $X^{r}$ is vacuously Maskin monotonic. Moreover, by changing the domain of the rule so that preferences are only defined over $X^{r}$, we can establish that no veto power is also vacuously satisfied. Thus, any such rule is Nash implementable.

\section{Step 1: A preference relation is completely determined by its restriction} to $\mathrm{X}^{\mathrm{r}}$.

Let $i \in N$ and suppose that $R_{i}, R_{i}^{\prime} \in \mathcal{R}_{i}$. We establish that $\left.R_{i}\right|_{X^{r}}=\left.R_{i}^{\prime}\right|_{X^{r}}$ if and only if $R_{i}=R_{i}^{\prime}$. There exists $u_{i} \in \mathcal{U}_{i}$ such that for all $\bar{x}, \bar{y} \in X^{r}, \bar{x} R_{i} \bar{y}$ if and only if $\int_{0}^{\infty} e^{-\delta_{i} t} u_{i}(\bar{x}(t)) d t \geq \int_{0}^{\infty} e^{-\delta_{i} t} u_{i}(\bar{y}(t)) d t$.

Order the elements of $X=\left\{x_{1}, \ldots, x_{m}\right\}$. For all $\bar{x} \in X$, define an element $\bar{x}^{*} \in X^{r}$ so that for all $i=0, \ldots, m-1$, if $t \in[i-1, i)$, then $\bar{x}^{*}(t)=x_{i}$, and otherwise, $\bar{x}^{*}(t)=$ $\bar{x}(t+m)$. Then it is obvious that for all $\bar{x}, \bar{y} \in X^{*}$.

$$
\int_{0}^{\infty} e^{-\delta_{i} t} u_{i}(\bar{x}(t)) d t \geq \int_{0}^{\infty} e^{-\delta_{i} t} u_{i}(\bar{y}(t)) d t
$$

if and only if

$$
\int_{0}^{\infty} e^{-\delta_{i} t} u_{i}\left(\bar{x}^{*}(t)\right) d t \geq \int_{0}^{\infty} e^{-\delta_{i} t} u_{i}\left(\bar{y}^{*}(t)\right) d t .
$$

This is enough to establish the result.

\section{Step 2: Defining the social choice rule on $\mathrm{X}^{\mathrm{r}}$.}

Define $\psi^{*}:\left.\mathcal{R}\right|_{X^{r}} \rightrightarrows X^{r}$ by $\psi^{*}\left(\left.R\right|_{X^{r}}\right)=\psi(R)$. Step 1 tells us that $\psi^{*}$ is well-defined. As $\psi$ realizes all alternatives, the range of $\psi^{*}$ is actually a subset of $X^{r}$. As for all $i \in N$ and all $\left.\left.R_{i}\right|_{X^{r}} \in \mathcal{R}_{i}\right|_{X^{r}},\left.R_{i}\right|_{X^{r}}$ has no maximal choice (this follows from the fact that for all $R_{i} \in \mathcal{R}_{i}, R_{i}$ is nonconstant), the no veto power condition of Maskin [5] is vacuously satisfied.

\section{Step 3: The new rule is Maskin monotonic.}

We show that $\psi^{*}$ is Maskin monotonic. This is done by showing that for all $i \in N$ and all $\left.R_{i}\right|_{X^{r}},\left.\left.R_{i}^{\prime}\right|_{X^{r}} \in \mathcal{R}_{i}\right|_{X^{r}}$, if $\left.R_{i}^{\prime}\right|_{X^{r}}$ is a monotonic transformation of $\left.R_{i}\right|_{X^{r}}$ at any $\bar{x} \in X^{r}$, then $\left.R_{i}^{\prime}\right|_{X^{r}}=\left.R_{i}\right|_{X^{r}}$. This then implies that the hypothesis of Maskin monotonicity is vacuously satisfied, and $\psi^{*}$ is thus Maskin monotonic. By Maskin's theorem, there exists a game form $(S, g)$ which implements $\psi^{*}$ on this restricted domain.

Thus, let $\bar{x} \in X^{r}$. Let $\left.\left.R_{i}\right|_{X^{r}} \in \mathcal{R}_{i}\right|_{X^{r}}$. Let $u_{i}$ be a utility index corresponding to $\left.R_{i}\right|_{X^{r}}$. The index $u_{i}$ is nonconstant by hypothesis. Next, let $\left.R_{i}^{\prime}\right|_{X^{r}}$ be any other preference relation, with corresponding utility index $u_{i}^{\prime}$. The indices $u_{i}$ and $u_{i}^{\prime}$ are not cardinally related. If they were, then $R_{i}$ and $R_{i}^{\prime}$ would be the same preference relation. 
Let $Y=\left\{z \in \mathbb{R}_{++}^{X}: \sum_{x \in X} z_{x}=\frac{1}{\delta_{i}}\right\}$. Any element of $X^{r}$ can be associated with an element of $Y$, and each element of $Y$ can be associated (nonuniquely) with an element of $X^{r}$.

Associate $\bar{x}$ with $z \in Y$ such that for all $x \in X, z_{x}=\int_{0}^{\infty} e^{-\delta_{i} t} 1_{\{\bar{x}(t)=x\}} d t$. Then, $U_{i}(\bar{x})=u_{i} \cdot z$ and $U_{i}^{\prime}(\bar{x})=u_{i}^{\prime} \cdot z$. Clearly, as each of $u_{i}$ and $u_{i}^{\prime}$ are nonconstant, and as $z$ is in the interior of the simplex, there exists $z^{\prime} \in Y$ such that $u_{i} \cdot z \geq u_{i} \cdot z^{\prime}$ and $u_{i}^{\prime} \cdot z^{\prime}>u_{i}^{\prime} \cdot z$. The vector $z^{\prime}$ can then be associated with an element of $X^{r}$ : simply let $\bar{x}^{\prime} \in X^{r}$ such that $\int_{0}^{\infty} e^{-\delta_{i} t} 1_{\left\{\bar{x}^{\prime}(t)=x\right\}} d t=z_{x}^{\prime}$. Then, $\left.\bar{x} R_{i}\right|_{X^{r}} \bar{x}^{\prime}$ and $\left.\bar{x}^{\prime} P_{i}^{\prime}\right|_{X^{r}} \bar{x}$. Thus, $\left.R_{i}^{\prime}\right|_{X^{r}}$ is not a monotonic transformation of $\left.R_{i}\right|_{X^{r}}$.

Thus, $\psi^{*}$ trivially satisfies Maskin monotonicity. We conclude that $\psi^{*}$ is Nash implementable.

\section{Step 4: The original rule is Nash implementable.}

To see that $\psi$ is Nash implementable, let $(S, g)$ be the game form which Nash implements $\psi^{*}$. We claim that it also Nash implements $\psi$. Let $R \in \mathcal{R}$. We claim that the Nash equilibria for the game induced by $\left(S, g,\left.R\right|_{X^{r}}\right)$ coincide with the Nash equilibria for the game induced by $(S, g, R)$. In particular, $g\left(N\left(S, g,\left.R\right|_{X^{r}}\right)\right)=\psi^{*}\left(\left.R\right|_{X^{r}}\right)$. By definition of $\psi^{*}, \psi^{*}\left(\left.R\right|_{X^{r}}\right)=\psi(R)$. If $s$ is a Nash equilibrium for $\left(S, g,\left.R\right|_{X^{r}}\right)$, then it is also a Nash equilibrium for $(S, g, R)$. To see this, let $i \in N$ and let $s_{i}^{\prime} \in S_{i}$. Then $\left.g(s) R_{i}\right|_{X^{r}} g\left(s_{i}^{\prime}, s_{-i}\right)$, so that by definition, as $g(s), g\left(s_{i}^{\prime}, s_{-i}\right) \in X^{r}, g(s) R_{i} g\left(s_{i}^{\prime}, s_{-i}\right)$, and $s$ is a Nash equilibrium for $(S, g, R)$. Similarly, it can be established that if $s$ is a Nash equilibrium for $(S, g, R)$, then it is also a Nash equilibrium for $\left(S, g,\left.R\right|_{X^{r}}\right)$. This establishes that $g(N(S, g, R))=\psi(R)$, so that in fact $\psi$ is Nash implementable by the game form $(S, g)$.

\section{Conclusion}

This paper contributes to the literature on implementation by pointing out a previously unrecognized connection between virtual and repeated implementation. Our model focuses on continuous time. Kalai and Ledyard's seminal work is done in discrete time. A result analogous to our main theorem in discrete time is therefore desirable.

\section{References}

[1] D. Abreu and A. Sen, Virtual implementation in Nash equilibrium, Econometrica 59 (1991), 997-1021.

[2] M. Jackson, A crash course in implementation theory, Social Choice and Welfare $\mathbf{1 8}$ (2001), 655-708. 
[3] E. Kalai and J. Ledyard, Repeated implementation, Journal of Economic Theory 83 (1998), 308-317.

[4] T. Koopmans, Stationary ordinal utility and impatience, Econometrica 28 (1960), 287-309.

[5] E. Maskin, Nash equilibrium and welfare optimality, Review of Economic Studies 66 (1999), 23-38.

[6] H. Matsushima, A new approach to the implementation problem, Journal of Economic Theory 45 (1988), 128-144. 